\title{
Abstracts presented by third-year residents at Research Day in 2019 at Texas Tech University Health Sciences Center Internal Medicine residency program in Lubbock, Texas
}

\section{Impact of ACEI/ARB drugs on Renal Function in Chronic Kidney Disease Patients undergoing Percutaneous Coronary Intervention \\ P Ratanasrimetha, Y Vorakunthada, T Mingbunjerdsuk, K Nugent}

Background: Chronic kidney disease (CKD) is one of the risk factors for coronary heart disease (CHD). Pharmacological intervention is part of the effective management for CHD, particularly with ACEI/ARB drugs, which provide a protective effect by preventing left ventricular remodeling. $\mathrm{PCl}$ is one of the most common interventions in these patients but can potentially lead to contrast-induced nephropathy, especially in high risk populations like CKD patients.

Methods: Single center retrospective cohort study conducted in chronic kidney disease patients undergoing $\mathrm{PCl}$. Study enrollment period is between January 1, 2015, and December 31, 2016, and follow up period until June 30,2017 . All participants need to take ACEI/ARB for at least 1 month prior to the $\mathrm{PCl}$.

Results: 83 patients were included in this study. Sixty-nine patients were in the continued drug group during hospitalization for catheterization, and 14 patients were in the discontinued drug group. We found a significant decrease in creatinine for the discontinued drug group day 1 post $\mathrm{PCl}, \mathrm{p}<0.02$. The mean percentage change for the discontinued drug group was $-12.2 \%$ and for the continued drug group it was $+1.2 \%$. The effect was consistent but not significant for day 2 , day 3, and 1-month post $\mathrm{PCl}$.

Conclusions: This study demonstrates a significant decrease in creatinine levels on day 1 post $\mathrm{PCl}$ in the discontinued drug group but no significant difference on day 2 , day 3 and 1 -month post PCl.

Corresponding author: Michael Phy

Contact Information: Michael.Phy@ttuhsc.edu DOI: 10.12746/swrccc.v7i30.569

\section{Prevalence of Bactericidal/Permeability- Increasing Protein Autoantibodies in Cystic Fibrosis Patients: Systematic Review and Meta-analysis}

K Iwuji, E Larumbe-Zabala, S Bijlani, K Nugent, A Kanu, E Manning, $X$ Solis

Background: There have been varying reports on the prevalence of antineutrophil cytoplasmic antibodies with bactericidal/permeability-increasing protein (BPIANCA) specificity in cystic fibrosis (CF) patients. These autoantibodies are believed to develop in response to infection and colonization, especially with Pseudomonas aeruginosa. The aim of this review was to estimate the overall prevalence of BPI-ANCA in CF patients.

Methods: We searched PubMed, Scopus, and Embase databases for studies reporting the prevalence of BPIANCA in CF patients. We also searched the Journal of Cystic Fibrosis and our institution's library for relevant studies on BPI-ANCA. We calculated the proportion with a $95 \%$ confidence interval (CI) to assess the prevalence of BPI-ANCA in the individual studies and then pooled the results using a random effects model. Publication bias was assessed using graphical and statistical methods. Finally, we assessed statistical heterogeneity using the $\mathrm{I}^{2}$ test.

Results: Our search yielded 12 eligible studies published between 1996 and 2015. The prevalence of BPIANCA in patients with CF varied from $17.9 \%$ to $83.0 \%$ with a pooled prevalence of $49.5 \%(95 \% \mathrm{Cl}$ $35.5 \%-63.4 \%$ ). No evidence of publication bias was found. However, there was evidence of statistically significant variation in the prevalence estimate due to heterogeneity $(I 2=93.4 \%, P<0.01)$.

Conclusions: Given the highly varying prevalence of BPI-ANCA in patients with CF, more welldesigned prospective clinical studies are needed to determine its true prevalence and clinical relevance.

In Press: Pediatric Allergy, Immunology, and Pulmonology. 


\section{Fellowship Match at Texas Tech University Internal Medicine Residency: What Factors Matter?}

\author{
S Suchartlikitwong, W Vutthikraivit, J Makrum, \\ M Phy
}

Background: The Internal Medicine residency program at Texas Tech University Health Sciences Center has trained excellent board-certified internists for more than 4 decades. This study aimed to analyze characteristics of residents in the Internal Medicine residency program at Texas Tech University Health Sciences Center who applied to fellowships and to compare characteristics of those who successfully matched and those who did not match.

Methods: An online web-based questionnaire was sent to all residents in post-fellowship-match periods in the years 2014 to 2018. Data collection included baseline characteristics, citizenship, medical schools, USMLE scores, subspecialties applied to, matching results, and travel expenses. Data were analyzed by SPSS software using Chi-square tests for categorical data and T-tests for continuous data.

Results: Fifty-four residents responded to the questionnaire, and $42(78 \%)$ residents had applied to fellowships. The match success rate on the first attempt was $84 \%$. There were no significant associations between citizenship, medical school graduation, USMLE scores, being a chief resident or a house staff officer, doing an away rotation, or the number of scholarly activities and the fellowship match success. Based on logistic regression, there were no individual factors that significantly predicted the match success.

Conclusions: In our study, there is no significant association between any characteristics of the residents and fellowship match success. The low number of participants in our study $(n=42)$ could contribute to the lack of power to detect statistically significant factors which might determine match success.

\section{Opportunities for Procedural Skills Among Medical Residents in Critical Care Setting in West Texas}

\section{Y Vorakunthada, W Lilitwat, M Phy}

Background: Competency in procedural skills is an integral part of Internal Medicine (IM) residency training. As part of the requirement for certification, the American Board of Internal Medicine (ABIM) requires demonstration of competency in procedural skills, and the number of procedures recorded reflects this. However, sufficient opportunities to perform procedures by residents in the critical care setting remain uncertain.

Objectives: To investigate procedures performed in a medium-sized academic program in Lubbock, Texas, identify deficiencies, and suggest recommendations.

Methods: We conducted a retrospective review of the electronic databases for all procedures logged by IM residents from the year 2013 to 2017 . Data were collected, coded, and analyzed using the SPSS program.

Results: The most common three procedures in our training program were central line placement (median 37, Q25-75: 28-48), arterial line placement (median 14, Q25- 75: 8-19), and orotracheal intubation (median 10, Q25-75: 6-15). Residents performed lumbar puncture, thoracentesis, and abdominal paracentesis only 2-3 times per year. Arthrocentesis was the least performed procedure in the program. Per year of training, PGY-3 residents tended to have fewer opportunities to perform procedures compared to the PGY-1 residents due to the nature of clinical rotations.

Conclusions: Procedural stimulation and dedicated procedural rotation might be an alternative for improving residents' skills, performance, and competency, especially for uncommon procedures like arthrocentesis. Simplifying the data entry process might improve compliance. The limitation of this study is its retrospective data collection, and residents might under-report their procedures in the system.

\section{Statins reduce the risk of clostridium difficile diarrhea: A meta-analysis}

S Suchartlikitwong, P Laoveeravat, J Teerakanok, T Mingbunjerdsuk, S Thavaraputta, W Vutthikraivit, C Thongprayoon, K Nugent, W Cheungpasitporn

Introduction: Clostridium difficile is the most common cause of infectious diarrhea in hospitalized patients. There has been emerging clinical evidence suggesting 
a protective effect of 3-hydroxy-3 methyl glutaryl (HMG)CoA reductase inhibitors, statins, against Clostridium difficile infection (CDI). The aims of this meta-analysis were to assess the risks of $\mathrm{CDI}$ and clinical outcomes of CDI in patients who received statins vs. no statins.

Methods: A literature search was performed using MEDLINE and EMBASE from their inception through May 2017. Studies that reported odds ratios or relative risks comparing the risk of developing $\mathrm{CDI}$ and/or the clinical outcomes of $C D I$ in patients who received statins treatment versus those who did not receive statins were included. Pooled odds ratios (OR) and 95\% confidence interval $(\mathrm{Cl})$ were calculated using a random-effect and generic inverse variance method.

Results: Ten observational studies (1 cohort study and 9 case-control studies with 37,109 patients) were analyzed. Compared to no treatment, statins reduced the risk of CDI development (6 studies; OR 0.66, 95\% $\mathrm{Cl} 0.44$ to 0.99 ). However, among patients who developed CDI, statins did not significantly reduce recurrent CDI risk (2 studies; OR $0.69,95 \% \mathrm{Cl} 0.28$ to 1.71 ) or 30 -day mortality (3 studies; OR $0.77,95 \% \mathrm{Cl} 0.51$ to 1.14). The data on severity of CDI in patients who used statins were limited. A study demonstrated an insignificant association between statins use and reduced severity of CDI (OR $0.85,95 \% \mathrm{Cl} 0.55$ to 1.32$)$ when compared with no statins use.

Conclusion: Our study demonstrates a significant association between statin use and reduced risk of CDI development. However, the findings of our study suggest no significant associations between statins use and improvement in clinical outcomes of $\mathrm{CDI}$, including recurrent CDI or 30-day mortality. These findings may impact clinical management and primary prevention of CDI.

Published: Proc (Bayl Univ Med Cent) 2018 Sep 19; 31(4):447-452.

\section{Pneumomediastinum: A focused review}

\section{Sara Mousa, Hawa Edriss}

Background: Pneumomediastinum(PM) is defined as the presence of abnormal gas in the mediastinum. Air leakage from ruptured alveoli into the mediastinum can occur spontaneously, or following trauma, invasive procedures, and thoracic surgery, or during invasive mechanical ventilation (IMV). Non-invasive ventilation (NIV) has also been reported to cause PM. Rupture of the alveoli leads to leakage of air along bronchovascular bundles into the hilum and from there into the mediastinum. Along with PM, pneumothorax, subcutaneous emphysema, and sometimes pneumoperitoneum are known complications associated with mechanical ventilation (MV) and are collectively called barotrauma. A decrease in lung compliance, e.g., acute respiratory distress syndrome (ARDS), age, and underlying lung diseases, such as interstitial lung disease (ILD), chronic obstructive pulmonary disease (COPD), cystic fibrosis, and certain lung infections like Pneumocystis jiroveci pneumonia (PJP), are risk factors for PM.

\section{Methods: focused literature review}

\section{Results and Conclusions:}

1. The incidence of PM in mechanically ventilated patients is higher in ARDS and has decreased with the low tidal volume ventilation.

2. Chest $x$-rays can miss up to $25 \%$ of small PMs detected by CT scans of the chest.

3. Pneumomediastinum is managed with low tidal volume ventilation with plateau pressures less than $30 \mathrm{~cm} \mathrm{H}_{2} \mathrm{O}$ and treatment of underlying lung disease. Novel ways of ventilation, such as high frequency oscillatory ventilation and asynchronous independent lung ventilation, may improve ventilation in some patients.

4. Spontaneous PM can often be managed as an outpatient unless the underlying cause needs medical treatment or further workup.

Published: Pneumomediastinum secondary to invasive and non-invasive mechanical ventilation. The Southwest Respiratory and Critical Care Chronicles 2019; v.7 (27):36-42.

7. Impact of Preoperative Atrial Fibrillation in Patients with Left Ventricular Assist Device: A Systematic Review and Meta-Analysis

Pakpoom Tantrachoti, Saranapoom Klomjit, Was awat Vutthikraivit, Sofia Prieto, Nandini Nair

Background: Atrial fibrillation (AF) is commonly found in patients being evaluated for left ventricular 
assist devices (LVAD). There are conflicting data regarding the mortality risk and the thromboembolic risk in patients with pre-operative $A F$ who undergo LVAD implantation. We examined these risks more in depth using meta-analysis.

Methods: A literature search of PubMed, EMBASE, SCOPUS, and Cochrane from inception to January 2018 was conducted. The eligible studies must compare mortality rate between AF and Non-AF (NAF) groups after LVAD implantation. The quality of all included studies was evaluated using the NewcastleOttawa Scale. Meta-analysis from the combined extracted data was conducted using the random effect, generic inverse variance method of DerSimonian and Laird. Meta-analysis of thromboembolic risk was also performed using the data from the included studies.

Results: We obtained 379 articles from our search strategy. Six retrospective studies were included and accounted for 5,297 LVAD patients (AF 1,360; NAF 3,937). The median follow-up duration ranges between 7-24 months. The pooled analysis revealed a significantly increased risk of mortality in pre-operative AF patients who underwent LVAD operation compared to those with NAF (Risk Ratio [RR] 1.19, 95\% Cl 1.07$1.33, \mathrm{I}^{2}=0 \%$ ). Sensitivity analysis was conducted to confirm the result. Four studies reported the thromboembolism events involving 1,130 pre-operative AF and 3,596 NAF patients. The pooled analysis did not show a statistically significant association between risk of thromboembolic event and pre-operative AF (RR 1.04, $\left.95 \% \mathrm{Cl} 0.75-1.43, \mathrm{I}^{2}=81 \%\right)$.

Conclusion: Our study shows that pre-operative AF may be associated with a higher mortality rate in the long-term period. However, the implication of this result is limited by the fact that the data are pooled from only retrospective studies. Despite this limitation, this study should encourage clinicians to consider $A F$ as a risk factor for poorer post-operative outcomes in patients undergoing LVAD surgery.

This work is licensed under a Creative Commons Attribution-ShareAlike 4.0 International License. 